\title{
Causas y efectos de la discriminación y la violencia de género en el ámbito universitario
}

\author{
Causes and effects of discrimination and gender violence in the university setting \\ Vanesa Vazquez Laba \\ Instituto de Altos Estudios (IDAES), Argentina Universidad Nacional de San Martín (UNSAM). \\ CONICET, Argentina \\ vanesavazquez.laba@gmail.com \\ Mariana Palumbo \\ Instituto Interdisciplinario de Género (IIEGE), Argentina Universidad de Buenos Aires (UBA). \\ CONICET, Argentina \\ mrnpalumbo@gmail.com
}

\section{ReSUMEN:}

La violencia simbólica es un tipo de violencia frecuente en el ámbito universitario debido a su naturalización entre los distintos actores de la comunidad universitaria. Esta es infringida mayoritariamente por los estudiantes varones contra las estudiantes mujeres. Sin embargo, los profesores varones y no docentes también practican violencias simbólicas y acoso a través de redes sociales, que se interseccionan con abusos de poder. En el siguiente artículo, describimos y analizamos los fundamentos y efectos de la violencia de género de tipo simbólica que vivencian las y los estudiantes de la Universidad Nacional de San Martín (UNSAM). El análisis de la violencia simbólica en estos vínculos será a partir de un abordaje cuantitativo y cualitativo. Las fuentes de las cuales nos valemos son, por un lado, los resultados de una encuesta desarrollada por la UNSAM y aplicada a la población estudiantil de las distintas unidades académicas; y por el otro, información concerniente a las denuncias y consultas recibidas por la Consejería de Atención a la Violencia de Género y Sexualidades sobre hechos de discriminación y violencia de género que padeció el alumnado dentro del ámbito universitario.

Palabras Clave: Violencia de Género, Vida Universitaria, Estudiantes, Relaciones de Género, Argentina.

\section{AbStraCt:}

Symbolic violence is a common type of violence because it is naturalized by various members of the university community. The majority of symbolic violence is inflicted by male students against female students. However, male professors and members of the non-academic staff also engage in symbolic violence and social media harassment acts involving abuse of power. The purpose of this article is to describe and analyze the causes and effects of symbolic gender violence experienced by male and female students at Universidad Nacional de San Martín, Argentina. The methodology used combines quantitative and qualitative approaches. Th e sources we utilized are, on one hand, the results of a survey developed and applied by the University of San Martin (UNSAM) to their students from different academic units; and, by the other hand, information regarding to denunciations and queries received by the Counseling for Gender and Sexuality Violence about facts of discrimination and gender violence suffered by students within the university context.

KeYwords: Gender Violence, College Life, Gender Relations, Students, Argentina.

\section{INTRODUCCIÓN}

En el año 2009, el Estado Argentino promulgó la Ley 26.485 de "Protección Integral para Prevenir, Sancionar y Erradicar la Violencia contra las Mujeres en los Ámbitos en que Desarrollen sus 
Relaciones Interpersonales". Dicha ley es una de las conquistas del movimiento feminista y de mujeres local en la puja por una mayor igualdad entre los géneros y una mejor convivencia de la sociedad toda. En el marco de este avance, en relación con la temática de violencia contra las mujeres, cuatro años más tarde, en el año 2013, a raíz de un doble femicidio de una estudiante y su madre, el Rector de la Universidad Nacional de San Martín (UNSAM) ${ }^{1}$ resolvió crear un espacio de investigación, formación, sensibilización y atención, asumiendo un compromiso institucional en la lucha por garantizar una universidad libre de violencias y que apunte al bienestar de la comunidad universitaria en su conjunto. En ese entonces, el Programa contra la Violencia de Género, hoy Dirección de Género y de Diversidad Sexual (DGyDS) ${ }^{2}$ interviene en casos de discriminación y violencia de género ${ }^{3}$ tanto de la propia universidad como provenientes de la comunidad del Partido de General San Martín y zonas aledañas. ${ }^{4}$

En los cinco años de experiencia de atención de casos de violencia de género, las situaciones de violencia simbólica, muchas veces atravesadas por otros tipos de violencia, son las más denunciadas por los distintos actores que intervienen en la Universidad. En el año 2016, la DGyDS (ex Programa contra la Violencia de Género) realizó un estudio denominado "Diagnóstico sobre Discriminación y Violencia” con la finalidad de conocer qué tipos de violencias de género prevalecen en la universidad. En dicha investigación se identificó que un $50 \%$ de los/as estudiantes encuestados dicen haber escuchado comentarios sexistas o discriminatorios sobre características, conductas o capacidades a causa de género u orientación sexual y un $11 \%$ dice haber sido descalificado o desvalorizados por estas causas.

A partir de este hallazgo, basado en que la violencia simbólica es la que más experimenta el estudiantado de la UNSAM, en el siguiente artículo nos proponemos describir y analizar los fundamentos y los efectos de este tipo de violencia. Partimos de la premisa de que las violencias de género se encuentran interrelacionadas, así, por ejemplo, la violencia psicológica tiene efectos físicos sobre las personas y se basa en sustentos simbólicos. ${ }^{6}$ No obstante, a los fines analíticos, examinaremos a la violencia simbólica en su especificidad en tanto aparece como el tipo de violencia más frecuente, según los testimonios de las denuncias y los datos de la encuesta.

En este artículo conceptualizamos a la violencia de género desde la perspectiva de Aponte Sánchez y Femenías (2009), la cual se enmarca dentro de la definición desarrollada en la Cuarta Conferencias Mundial sobre la Mujer de 1995. ${ }^{7}$ Para estas autoras la violencia de género son "aquellos actos o amenazas, sea en el hogar o en la comunidad, incluyendo los actos perpetrados o tolerados por el Estado, que infunden miedo e inseguridad en la vida de las mujeres e impiden lograr la igualdad, el desarrollo y la paz" (Aponte Sánchez y Femenías, 2009, p. 44).

Definimos a la violencia simbólica acorde a la ley 26.485, en la cual subyacen los aportes pioneros de las autoras antes citadas. En la misma se establece que este tipo de violencia es aquella que "a través de patrones estereotipados, mensajes, valores, íconos o signos transmita y reproduzca dominación, desigualdad y discriminación en las relaciones sociales, naturalizando la subordinación de la mujer en la sociedad". 8 Consideramos que la reiteración de casos de violencia simbólica se debe a que es la violencia más naturalizada y posee mayor legitimidad social. La violencia simbólica, para Aponte Sánchez y Femenías (2009), funciona como legitimadora de la violencia, ya que permite que la misma sea percibida dentro de los cánones de la normalidad. Para ellas, la violencia simbólica impone un orden bajo el supuesto de que es inmodificable e incuestionable, por lo que ese orden se funda en la ética, la moral y las costumbres de una sociedad dada. Entendemos que las violencias psicológica y simbólica son violencias que aunque más sutiles no son menos efectivas sobre los sujetos que las vivencian.

El análisis de la violencia simbólica en estos vínculos se llevó a cabo partir de un abordaje cuantitativo y cualitativo. Las fuentes de las cuales nos valemos son, por un lado, los resultados de la encuesta aplicada a la población estudiantil de las distintas unidades académicas, ${ }^{9}$ ubicadas en el campus de la UNSAM y la Escuela de Economía y Negocios localizada en el centro del partido de San Martín. ${ }^{10}$ Las encuestas fueron realizadas por encuestadores/as quienes habían sido capacitados/as previamente. Las mismas eran 
de carácter voluntario, anónimas y se le entregó a cada persona encuestada un consentimiento informado donde constaban datos de contacto del Programa contra la Violencia de Género. Las mismas tuvieron lugar en espacios de común acuerdo, principalmente se llevaron a cabo dentro del campus.

En relación con las dimensiones que fueron relevadas, la encuesta se dividió en cuatro apartados: 1) los datos sociodemográficos de las personas encuestadas, el objetivo de esta sección de la encuesta fue establecer las características sociodemográficas de la población estudiada (edad, género autopercibido, si tenían hijos, si trabajaban, lugar de residencia), cuánto tiempo hacía que cursaban y en qué año de la carrera se encontraban; 2) violencia de género, este apartado indagó sobre las representaciones y experiencia de violencia de género y discriminación en distintos ámbitos de sociabilidad, a saber hogar, trabajo, vía pública y transporte, universidad, en instituciones de salud, entre otros; 3 ) violencia en los vínculos sexuales o de pareja (formales e informales), esta sección examinó si las personas encuestadas experimentaron o perpetraron violencia dentro de estos tipos de relaciones y cuál es el género de quien la ejerció. Asimismo, se consultó sobre qué hicieron ante estas situaciones y se abordaron sus representaciones sobre el amor romántico y la violencia; por último, 4) violencias dentro de la universidad, en este apartado se consultó sobre situaciones de violencia de género y de discriminación dentro del ámbito universitario y sobre las jerarquías de las personas que las perpetraron. También, se examinó cuáles creían, las personas encuestadas, que eran las medidas que debería tomar la Universidad frente a las mismas, entre otras cuestiones afines.

Respecto a la metodología cualitativa se utilizó información concerniente a las denuncias y consultas recibidas por la Consejería de Atención a la Violencia de Género y Sexualidades sobre hechos de discriminación y violencia de género que padeció el alumnado dentro del ámbito universitario. ${ }^{11}$ Estas fueron analizadas de manera anónima y confidencial.

\section{La Universidad Nacional de SAN Martín SE PIEnSa}

El ejercicio legítimo de la violencia por razones de género ha sido confiscado a lo largo de la historia al ámbito privado; la literatura precursora sobre este tema comenzó develando el maltrato, la violación en los vínculos de pareja y familiares, es por ello que las primeras herramientas legales y la terminología se circunscribió a la violencia denominada familiar/doméstica. La distinción entre este ámbito y el público ha sido y continúa estando en debate. Según Brown (2009), quien retoma a Bobbio (1985), para la tradición liberal el espacio privado es aquel en el que las personas se realizan plenamente, mientras que, para la republicana el espacio de realización plena es el público. La sexualidad y la violencia, desde la perspectiva liberal, pertenecen al ámbito íntimo y privado. Por el contrario, para la republicana, éstos son asuntos que se dirimen en el ámbito público mediante la aplicación de políticas sexuales y, sobre todo, de políticas demográficas concretas (Brown, 2009).

Para Brown, quien se basa en esta lectura dicotómica, resulta que al derecho público le corresponde la ley, en cambio, al derecho privado lo rige la fórmula del contrato (privado): "El contrato rige los acuerdos entre particulares, mientras que la ley rige los acuerdos comunes y generales sujetos a coacción estatal” (Brown, 2009 , p. 24). Es decir, por ejemplo, el contrato matrimonial, que es un acuerdo entre particulares, es lo que opera como marco regulatorio de las relaciones de pareja y de la calidad de estos vínculos. De allí, si se mira desde una perspectiva liberal, el Estado no debería intervenir regulando prácticas que considera "íntimas". Sobre este fundamento se basa la idea de que las instituciones universitarias deben encargarse exclusivamente de las cuestiones concernientes a lo académico y al conocimiento y no a los vínculos interpersonales.

No obstante, los datos que se han recolectado marcan que la cotidianeidad de las interacciones entre los sujetos en este ámbito y la forma en la cual se produce conocimiento está atravesada por componentes heteronormativos y atributos de la masculinidad hegemónica (Connell, 1995; Massey, 1994), lo cual devela prácticas de violencia simbólica tales como desvalorización, subestimación y discriminación por la condición sexual o identidad de género. En este sentido, explica Rafael Blanco: 
en la universidad nos constituimos como cuerpos colectivos: académicos, estudiantiles, científicos, docentes, y también políticos, partidarios, gremiales, disciplinares, colegiados. Estudiamos, enseñamos e investigamos a partir de corpus textuales, de saberes, teorías, disciplinas, campos de conocimiento y tradiciones. Finalmente, como cuerpos racializados, genéricos y sexuados, atravesamos y conformamos el espesor de la vida universitaria en los lazos cotidianos (Blanco, 2016, p. 3).

El autor es claro en esta afirmación sobre las diferencias dentro del colectivo estudiantil y, por lo tanto, sobre la importancia de la convivencia en la igualdad. Por el contrario, los datos relevados reflejan un clima de permanente discriminación frente a las diferencias, las de orientación sexual e identidad de género, y fundamentalmente contra las mujeres.

Como observamos a continuación, las situaciones de violencia afectan e intervienen no sólo en la cotidianeidad de los sujetos directamente involucrados sino, también, de todo el entorno y de la institución universitaria en su conjunto. Por ejemplo, el 49\% de lxs estudiantes relevados en la encuesta dice que en el ámbito universitario son frecuentes los chistes y las burlas referidas a la sexualidad y al género (ver Figura 1).

\section{FIGURA 1}

¿Son frecuentes en el ámbito universitario los chistes, burlas, sarcasmos que aluden a ser varón, mujer, trans, u otro género, orientación sexual y/o identidad de género?

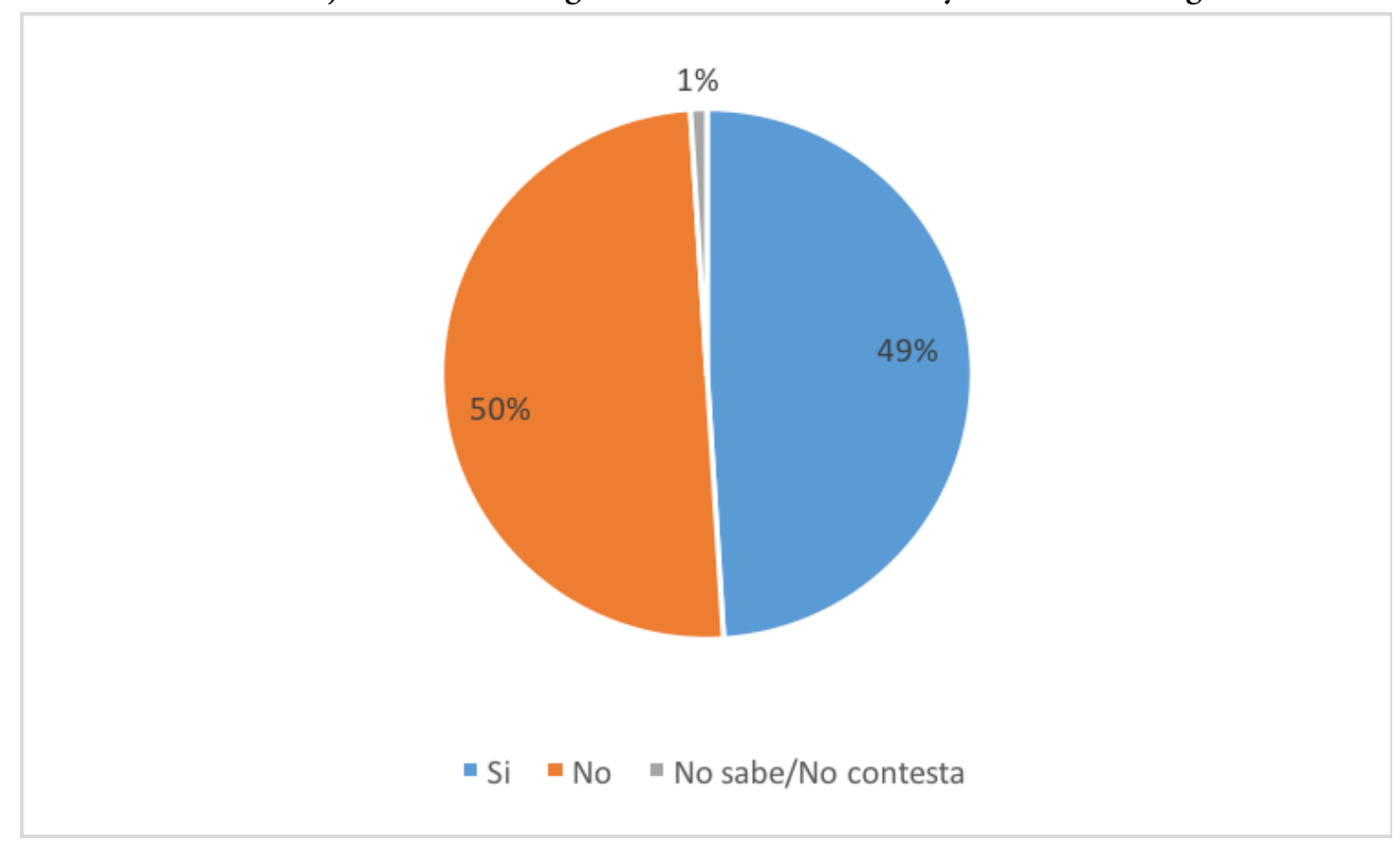

Fuente: elaboración propia

La DGyDS, a partir de cursos de capacitación, materias de grado y campañas de sensibilización sobre la temática, trabaja en pos del bienestar general y en contra de las situaciones de violencia de género o de cualquier otro tipo. Esto es reconocido por la población estudiantil: según los datos de la encuesta, la DGyDS es un espacio conocido por los/las estudiantes en un $45 \%$ de los casos. Si bien aún falta un mayor trabajo de posicionamiento institucional -entendido como una mayor sensibilización por parte de todos los claustros -, a través de los resultados de la encuesta podemos observar que su desempeño, en tanto espacio de ayuda ante situaciones de violencia de género y por orientación sexual, es ponderado positivamente por las/los estudiantes. Cuando se le preguntó al estudiantado, dentro de distintas opciones (podían elegir más de una), a quiénes le solicitarían ayuda o consejo frente a situaciones de discriminación, hostigamiento o abuso de género dentro de la UNSAM, la DGyDS fue el espacio el espacio mencionado en mayor medida, en un 54\%. 
Las otras dos instancias a las cuales les solicitarían ayuda o consejo son vínculos afectivos cercanos, amigxs, en primer lugar, y familia, en segundo lugar (ver Figura 2).

FIGURA 2

Frente a una situación de discriminación/hostigamiento/ abuso de género dentro de la universidad, ¿A quién/es le pedirías o pediste ayuda o consejo?

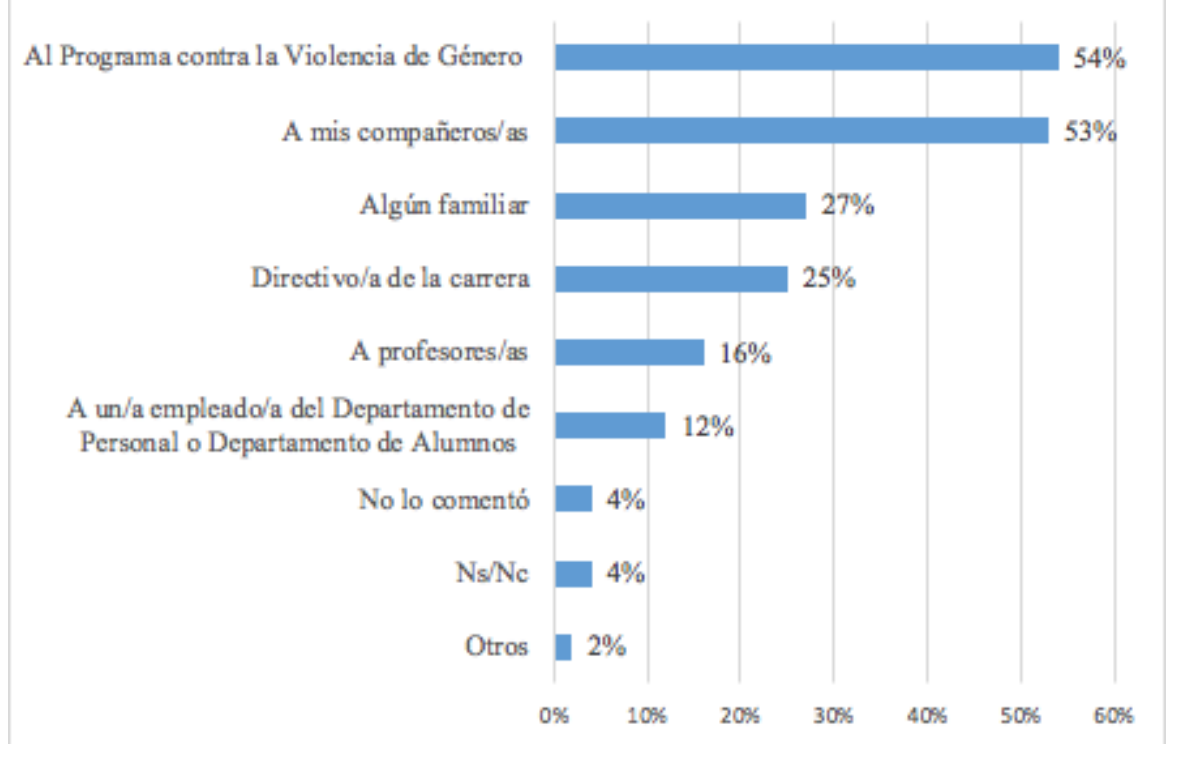

Fuente: elaboración propia

Esto permite pensar dos cuestiones, por un lado, que la DGyDS se posicionó rápidamente en tan sólo tres años (recordemos que la encuesta se realizó en el año 2016) - aunque aún falta que sea conocido en mayor medida por toda la población estudiantil- como un espacio efectivo para hacer frente a las denuncias y dar contención dentro de la institución. Sabemos que en otras instituciones los procesos se han dado de forma diferente, muchas veces con bastantes dificultades para resolver los casos y por eso se han tomado medidas alternativas como los "escraches" en las redes sociales por parte del estudiantado organizado. ${ }^{12}$ Por el otro, la creación del Programa contra la Violencia de Género en la UNSAM en el 2014 y el hecho de que las y los estudiantes lo reconozcan y consideren como un ámbito óptimo para acercar sus inquietudes y denuncias sobre discriminación y el acoso a causa de género y orientación sexual, en detrimento a hacerlo con sus vínculos afectivos directos (compañerxs y familiares), es un indicio de que la sexualidad ya no es vista meramente como un tema privado. Tal como explica Blanco (2016), se ha instalado dentro del imaginario colectivo de la universidad que la vida universitaria se conforma en sus lazos cotidianos entre sujetos racializados, genéricos y sexuados. En otras palabras, ha habido un corrimiento de la dicotomía público y privado, en términos de la perspectiva liberal (Bobbio, 1985).

Consideramos que otra de las herramientas que ha posicionado a la DGyDS como un interlocutor válido y reconocido dentro de la universidad es la generación de instancias burocráticas e institucionales de acción frente a la violencia de género y discriminación por orientación sexual. Además de las líneas de prevención y contención, se elaboró y aprobó el "Protocolo para la actuación en Situaciones de Discriminación y/ o Violencia de Género de la Universidad Nacional de San Martín”. ${ }^{13}$ Esto permite, por un lado, la visibilización de la temática; y por el otro, hacer sentir al alumnado contenido ante eventuales problemáticas de violencia. Estos hechos van en sintonía con las ideas del feminismo jurídico que considera al derecho y a los instrumentos legales como pasos iniciáticos en la construcción de la igualdad de género (Costa, 2016).

La UNSAM se convierte, así, en la cuarta universidad del país que tiene protocolo junto a las Universidades Nacionales del Comahue (2014), de La Plata (2015) y de Córdoba (2015). ${ }^{14}$ Vale recalcar que, aunque ha 
habido un avance importante desde el 2014 en la cantidad de universidades que comenzaron a adoptar un sistema institucional para el trabajo contra la violencia de género siguen siendo una pequeña proporción en relación al universo, representan sólo el $15 \%$ de las universidades nacionales. ${ }^{15}$ Estas cifras, también analizadas por Rovetto y Figueroa (2017), nos dan la pauta que aún persisten resistencias por parte de las instituciones a repensarse en su complejidad y atravesadas por múltiples vínculos y dimensiones que exceden lo estrictamente académico.

Estas propuestas se basan en la idea de que el "bien común" es fundamental como horizonte de trabajo dentro de las instituciones educativas. Subestimar que la violencia de género obstaculiza el buen desarrollo de la vida universitaria es hacer ojo miope a un problema que no sólo involucra a las mujeres o a quienes no se corresponde con los cánones de la masculinidad hegemónica, sino a la sociedad en general. Un ejemplo de un avance en este sentido, en otras latitudes, ha sido el fallo realizado por el Tribunal Superior de Justicia de Andalucía en el Estado español, en el cual se concluyó que "los delitos de violencia de género afectan a la seguridad pública en cuanto suponen una amenaza o atentado grave a un interés fundamental de la sociedad, cuál es el de proteger la dignidad e integridad física y moral de las personas que la integran (...)". ${ }^{16}$ En el caso argentino, tenemos la recientemente aprobada "Ley Micaela" (diciembre de 2018) la cual estipula la creación de un "Programa Nacional Permanente de Capacitación Institucional en Género y Violencia contra las Mujeres" con el objetivo de capacitar y sensibilizar a todos/as los/as funcionarios/as públicos, que integran los diferentes niveles del Estado. ${ }^{17}$ Este paso es fundamental ya que junto a la ley 26.485 de "Protección Integral para Prevenir, Sancionar y Erradicar la Violencia contra las Mujeres en los Ámbitos en que Desarrollen sus Relaciones Interpersonales”, establecen los criterios de carácter de urgencia frente al fenómeno de la violencia contra las mujeres y la responsabilidad del Estado en su tratamiento y prevención.

En dicho documento se destaca que este tipo de delito afecta los derechos fundamentales de las personas y que es responsabilidad del Estado la protección del derecho a vivir una vida a salvo de la violencia. Lo mismo se debe sostener para las instituciones educativas universitarias, estas deben garantizar los derechos de las/os estudiantes, docentes y no docentes en materia de violencia de género.

\section{LA EXPERIENCIA ESTUDIANTIL EN LA UNIVERSIDAD}

En este apartado, presentamos los datos que han sido recolectados por la propia DGyDS sobre las situaciones de violencia simbólica que viven las/os estudiantes a partir de dos fuentes primarias de información: por un lado, la encuesta "Diagnóstico sobre Discriminación y Violencia” y por otro lado, los relatos de las personas que han pedido asesoramiento y hecho denuncias.

Cuando se le consultó al estudiantado sobre diversas situaciones de violencia experimentadas a causa de género u orientación sexual, la mayor frecuencia se ubicó en las preguntas referidas a la violencia de tipo simbólica, a saber, gritos, burlas, comentarios y desvalorizaciones a causa de género y orientación sexual. Ante la pregunta "¿Has escuchado en la universidad comentarios sexistas (o discriminatorios) sobre las características, conductas o capacidades de mujeres, varones o trans?”, la respuesta fue afirmativa en un $50 \%$. En la misma línea, las personas encuestadas han sido descalificadas, burladas y desvalorizadas por género u orientación sexual en un $11 \%$.

Por su parte, la violencia de género de tipo física y sexual aparece en la encuesta aunque en valores mínimos. ${ }^{18} \mathrm{Si}$ bien no se registraron casos de violencia sexual con penetración, ${ }^{19}$ se identificaron, en un $4 \%$, escenas de violencia sexual y física sin penetración, a saber de estudiantes que fueron forcejeados/as y besados/ as sin su consentimiento.

En relación con quiénes realizaron estas prácticas violentas, las situaciones de violencia simbólica que experimentó el estudiantado fueron perpetradas por personas que ocupan distintas posiciones académicas dentro de la institución, a saber, estudiantes, docentes y no docentes. 
Respecto de los comentarios sexistas o discriminatorios sobre las características, conductas o capacidades de mujeres, varones o trans, los mismos fueron realizados por el estudiantado en un 54\%, por el plantel docente en un $6 \%$, y por los no docentes en un $2 \%$. Asimismo, hay casos en los cuales los comentarios fueron efectuados tanto por docentes como estudiantes en un $26 \%$; por estudiantes y no docentes en un $8 \%$; y por estudiantes, docentes y no docentes en un $4 \%$.

También las burlas, gritos, desvalorizaciones o bromas fueron perpetrados por distintos actores de la comunidad universitarias. En un 61\%, lo ejercieron estudiantes; en un 6\% docentes; en un 17\%, no docentes y en un $6 \%$, el estudiantado afectado dice que la violencia fue ejercida tanto por estudiantes, docentes y no docentes.

Aunque estas situaciones son mayormente perpetradas entre pares, es importante recalcar que el impacto es diferencial según quién la ejerza. En las clases artísticas, a partir de los testimonios de estudiantes mujeres a la DGyDS, se visualiza que algunos docentes varones utilizan frecuentemente como estrategia de enseñanza realizar comentarios sexistas a sus estudiantes mujeres. Por ejemplo, en una situación, el docente le pidió a una estudiante "que sea más perra", en un sentido sexual del término, para que logre una determinada caracterización de un personaje en una escena que no poseía en su guión ningún componente sexual. Esta situación está signada por un abuso de poder con connotaciones sexuales por parte del docente, dado que él coerciona a la estudiante en tanto que se posiciona como quien posee la técnica actoral adecuada para enseñarle y como quien tiene el poder de aprobarla académicamente o no. Si ella no actúa de la manera que él le marca, su desempeño será evaluado negativamente. Asimismo, estas situaciones de violencia simbólica son invisibilizadas y avaladas por el resto del estudiantado dado que se presupone que es el docente quien posee la autoridad y el conocimiento dentro del espacio aula.

Por su parte, las violencias físicas y sexuales cara a cara que no implican penetración, a saber, besar o tocar a una compañera sin su consentimiento, aparecen perpetradas sólo por estudiantes, entre pares. Sin embargo, se visualiza a los docentes y no docentes, al igual que a los estudiantes, acosando a estudiantes a través de redes sociales y medios digitales.

Respecto al acoso por redes sociales, ante la pregunta “¿Por parte de quién has recibido llamadas telefónicas, mensajes de texto, correos electrónicos o mensajes o comentarios a través de redes sociales referidos a tu vida íntima?", las personas encuestadas responden que en un $67 \%$ de los casos fue por parte de estudiantes, en un $13 \%$, por parte de docentes y en un $20 \%$, por parte de no docentes. A partir de los testimonios que recibimos de quienes se acercan a consultar o a denunciar a la Consejería integral de Atención a la Violencia de Género y Sexualidades aparece que estas formas de violencia, aunque mediatizadas por la tecnología, tienen efectos sobre el desarrollo académico y la autoestima de quienes las padecen, en su mayoría estudiantes mujeres.

En relación con el género autopercibido de los sujetos que experimentaron situaciones de violencia simbólica, según el género de quien la ejerció, se destacan los varones como los máximos perpetradores. Las mujeres sufren gritos, burlas y descalificaciones por parte de varones en un $67 \%$, tanto por parte de varones como de mujeres en un $28 \%$ y sólo por parte de otras mujeres en un $6 \%$. Asimismo, los varones experimentaron violencia simbólica por gritos, burlas y descalificaciones en un $22 \%$ de los casos. La misma fue perpetrada por parte de otros varones en un $6 \%$ y tanto por varones como por mujeres en un $17 \%$. Esto nos permite problematizar que, si bien la literatura sobre la temática ha marcado que el abuso de poder en las universidades es más frecuente por parte de los varones (Valls, Flecha y Melgar, 2008; Aguilar, Alonso, Melgar y Roldán, 2009), en la encuesta también se destaca que hay mujeres en una posición de poder perpetrando violencia simbólica sobre otras personas. Este punto es interesante destacar en el sentido de que, si bien no es considerado violencia de género el abuso de poder por parte de las mujeres, existen manifestaciones hostiles, de destrato, descalificaciones y maltrato perpetradas desde el ejercicio de la docencia que deben ser atendidas e investigadas.

Respecto a si los/as encuestados/as escucharon en la universidad comentarios sexistas o discriminatorios por género, orientación sexual o identidad de género, los varones encuestados son los mayores perpetradores 
en un $42 \%$ y las mujeres quienes más la sufren, en un 56\%. También, una mujer trans encuestada recibió comentarios sexistas y discriminatorios a causa de su identidad de género por parte de un varón. No obstante, los varones experimentaron este tipo de violencia en una alta proporción, aunque más baja que las mujeres, en un $42 \%$. La misma fue ejercida en un $20 \%$ por parte de otros varones y en un $22 \%$ por parte tanto de otros varones como de mujeres.

En relación con el acoso a través de redes sociales, los varones son quienes mayormente lo efectúan en un $80 \%$, mientras que las mujeres la realizan en un $20 \%$ de los casos. Por último, el tipo de violencia física y sexual de besar a una compañera sin su consentimiento, aparece perpetrada en un $71 \%$ de los casos por varones contra mujeres y en un $14 \%$ por parte de una mujer contra un varón. Es decir, si bien las mujeres son las que menos ejercen violencia y los varones son los mayores perpetradores, aparecen las mujeres efectuando prácticas violentas (Osborne Verdugo, 2009).

En resumen, en términos generales la violencia simbólica es un tipo de violencia frecuente y naturalizada por los distintos actores de la comunidad universitaria, más que otro tipo de violencia como, por ejemplo, la física o sexual. Consideramos que los gritos, insultos y descalificaciones entendidas como violencia de género, es decir, cuando tienen una connotación sexual, son formas de disciplinar a aquellas personas que no se adecuan a la heterosexualidad obligatoria (Rich, 1983) y a las mujeres y varones que no se corresponden con modelos de masculinidad y feminidad predefinidos (Rubin, 1993).

Dentro sus especificidades, la violencia simbólica se ejerce primordialmente entre el estudiantado. Los estudiantes varones son quienes perpetran mayoritariamente violencia simbólica sobre otras estudiantes mujeres. No obstante, los docentes y no docentes varones también practican violencias simbólicas y acoso a través de redes sociales, que se interseccionan con abusos de poder. Por último, aunque, en menor medida, las mujeres ejercen este tipo de violencia.

\section{Reflexiones finales para dar respuesta a la Violencia de género dentro de la UNIVERSIDAD}

Como se ha evidenciado en la encuesta, la creación y puesta en marcha de un espacio de Consejería dentro de la UNSAM, que realiza asistencia y contención a las personas que hayan sufrido una situación de discriminación y/o violencia por razones de género, es fundamental. El estudiantado considera como un interlocutor válido, con un $54 \%$, dicha política institucional.

Tal como se explicó en el artículo, cada vez son más las universidades nacionales que llevan adelante diferentes experiencias de abordaje frente a las situaciones de discriminación y/o violencia de género, ancladas muchas de éstas en las Secretarías de Extensión Universitaria o Bienestar Estudiantil. En el caso de la UNSAM, la DGyDS, como ya mencionamos, pertenece a la órbita del Rectorado, lo que asegura la transversalidad institucional de una política de género. Asimismo, es importante resaltar la existencia de interés político en la creación y ejecución de estas políticas con una asignación de recursos económicos y humanos, para poder generar así una real transversalidad institucional de políticas de géneros.

Una herramienta para poder intervenir, generar conciencia crítica y política feminista dentro de las instituciones es tener datos, cuantitativos y cualitativos, que permitan dimensionar las interacciones sociales, sexualizadas y generizadas, que existen en un espacio que se lo piensa a priori como meritocrático y desexualizado. Fue a partir de esta encuesta que observamos que el tipo de violencia que predomina en la universidad es la simbólica, a saber, situaciones de burla, chiste, denigración y subestimación son las formas en que se da la violencia que circula por los pasillos, las aulas y los claustros. Como sostienen Aponte Sánchez y Femenías (2009), esta misma violencia simbólica es la que legitima el orden de la diferencia y jerarquización entre los géneros intra claustro.

Los estudios evidencian que la única forma de hacerle frente a este tipo de actitudes es a través de la sensibilización y formación en igualdad de género. Es fundamental, visibilizar y hacer tomar conciencia de 
cómo la diferencia sexual se convierte en jerárquica y desigual, y, por lo tanto, es estructural. La convivencia intra claustro e inter claustro es un desafío importante si se piensa en universidades inclusivas y más democráticas.

La UNSAM, como ya se expresó en el cuerpo del texto, viene manifestando voluntad política frente a la prevención y erradicación de la violencia de género con todas las líneas de trabajo que se han implementado desde la DGyDS. Un último paso ha sido la aprobación en el último Consejo Superior del documento "Compromiso ético para las funciones de docencia", el cual establece principios éticos en el vínculo docenteestudiante. El mismo se empezará a implementar a partir de este año 2019. Erradicar la violencia de género en todas sus formas es prioritario para empezar a construir el bien común. Establecer códigos de convivencia basados en el respeto y la valoración mutua entre géneros es nuestro norte. Las herramientas que se han elaborado hasta el momento tales como los protocolos y dispositivos de atención deben ser medidas protectivas complementarias con un trabajo de formación y sensibilización permanente.

En definitiva, es indispensable tener en cuenta que, si se quiere pensar y/o prevenir la violencia de género desde las instituciones públicas, se debe abordar integralmente la problemática y avanzar por sobre los enfoques punitivistas o estigmatizantes centrados en la práctica del escrache o simple escarnio público que no dan respuestas acordes a las desigualdades y violencias existentes contras las identidades y corporalidades femeninas. Estas perspectivas no colaboran en la convivencia institucional universitaria, con lo cual, la UNSAM apuesta a las políticas preventivas y pedagógicas en un camino de cambio cultural profundo, pero, a su vez, atendiendo la contingencia de la problemática de la violencia con las medidas sancionatorias que habilita la normativa universitaria.

Esto es una invitación a la comunidad académica a poner en cuestión los fundamentos mismos de los paradigmas con que se ha estudiado la violencia de género, así como participar activamente del debate sobre cuáles son los dispositivos que pueden crearse que apunten a prácticas preventivas y a una transformación de los vínculos, reconociendo sus limitaciones, en pos del máximo bienestar de la comunidad universitaria.

\section{ReFERENCIAS}

Aponte Sánchez, E. y Femenías, M. L. (2009). Articulaciones sobre la violencia contra las mujeres. La Plata: Edulp.

Aguilar, C.; Alonso Olea, M. J.; Melgar Alcatud, P. y Roldán, S. (2009). Violencia de género en el ámbito universitario. Medidas para su superación. Pedagogía social: revista interuniversitaria, 16, 85-94.

Blanco, R. (2016). Más allá de los protocolos contra las violencias de género. Bordes: revista de política, derecho y sociedad, 3, 9-14.

Bobbio, N. (1985). Estado, gobierno y sociedad: por una teoria general de la politica. México D.F.: FCE.

Brown, J. (2009). Derechos (no) reproductivos y sexuales en los bordes entre lo público y lo privado. Algunos nudos del debate en torno a la democratización de la sexualidad. Sexualidad, salud y sociedad: revista latinoamericana, 2, 10-28.

Connell, R. (1995). Masculinities. Berkeley: University of California Press.

Costa, Malena (2016). Feminismos jurídicos. Buenos Aires: Ediciones Didot.

Massey, D. (1994). Space, place and gender. Minnesota: University of Minnesota.

Osborne Verdugo, R. (diciembre 2009). Construcción de la víctima, destrucción del sujeto: el caso de la violencia de género. Ponencia presentada en Jornadas Feministas de Granada, España. Recuperado de: http://www.feministas.org/ IMG/pdf/Mesa_Violencia_Raquel_Osborne.pdf

Rich, A. (1983). Sobre mentiras, secretos y silencios. Barcelona: Icaria.

Rovetto, F. y Figueroa, N. (2017). "Que la universidad se pinte de feminismo" para enfrentar las violencias sexistas. Descentrada, 1(2), Recuperado de: https://www.descentrada.fahce.unlp.edu.ar/article/view/DESe026/8707 
Rubin, G. (1993). Thinking sex: notes for a radical theory of the politics of sexuality. In Abelove, Henry; Barale, Michèle and Halperin, David (Ed.). The lesbian and gay studies reader (pp. 143-179). New York: Routledge.

Valls, R.; Flecha, A. y Melgar, P. (2008). Violència de gènere a les universitats catalanes: mesures per a la prevenció i superació. Temps d'educació, 35, 201-216.

\section{Notas}

1. Argentina. Poder Legislativo. (11 de marzo de 2009). Ley de Protección Integral para Prevenir, Sancionar y Erradicar la Violencia contra las Mujeres en los Ámbitos en que Desarrollen sus Relaciones Interpersonales [Ley 26.485].

2. La UNSAM se encuentra en el Partido de General San Martín, Provincia de Buenos Aires, Argentina. Este Partido limita con la Ciudad Autónoma de Buenos Aires, capital del país. El Campus de la Universidad ocupa actualmente un predio de 8,5 hectáreas, con $48.000 \mathrm{~m} 2$ de superficie y un plan total que alcanza los $220.000 \mathrm{~m} 2$ cubiertos.

3. En el año 2018 el Programa contra la Violencia de Género de la UNSAM pasó a ser DGyDS en la Secretaría Académica de la UNSAM.

4. Si bien retomamos la definición de violencia de la ley, la DGyDS no sólo piensa la violencia de género contra las mujeres sino también la violencia ejercida por connotaciones sexuales y que incluye a todos aquellos cuerpos que no se adecuan a la masculinidad hegemónica (Connell, 1995). La misma existe en tanto hay subordinación de otros grupos, que pueden ser las mujeres en su multiplicidad, pero también los hombres no heterosexuales, ciertos heterosexuales que no cumplen con los estereotipos esperados de masculinidad, o varones de color.

5. Las autoras de este artículo trabajan en la DGyDS de la UNSAM.

6. Entendemos por violencia psicológica a la que causa daño emocional y disminución de la autoestima, o perjudica y perturba el pleno desarrollo personal, o busca degradar o controlar a la mujer en sus diferentes prácticas; incluye la coerción verbal.

7. La Cuarta Conferencia Mundial sobre la Mujer tuvo lugar en la ciudad de Beijing, capital de la República Popular China, en septiembre de 1995.

8. Aquí, tal como se explicó en la introducción, ampliamos a las personas que no se corresponden con la masculinidad hegemónica.

9. Acorde a la población universitaria se generó una muestra representativa en proporción a la cantidad de población que poseen cada una de estas unidades académicas. La muestra es un estimativo del 1,5\% de los 11.688 del total de estudiantes para el año 2013. Esto se tradujo en 171 casos a ser relevados. Encuesta en el marco del proyecto PUENTE/UNSAM "Relaciones de discriminación y violencias basadas en el género en la Universidad Nacional de San Martín".

10. La UNSAM posee distintas unidades académicas e institutos de investigación tanto fuera como dentro del campus universitario, en el centro de la Ciudad de Buenos Aires como dentro del Partido de San Martín y otras ciudades del país, como por ejemplo Chascomús. A los fines de esta investigación se encuestaron a estudiantes que concurren al campus Miguelete con excepción de la dependencia de Economía y Negocios, con gran caudal de alumnado, la cual se ubica en el centro de San Martín. Las unidades académicas relevadas fueron la Escuela de Humanidades, Escuela de Economía y Negocios, Escuela de Política y Gobierno, Escuela de Ciencia y Tecnología, Arquitectura, Diseño y Urbanismo, Ciencia y Tecnología; Humanidades; Instituto de Altos Estudios Sociales; Instituto del Transporte; y el Instituto de Artes Mauricio Kagel. El desarrollo de la muestra se hizo en base a estas unidades académicas. Para ponderar la cantidad de estudiantes a encuestar por unidad académica se trabajó con los datos del Anuario 2013 "Oferta Académica y Población estudiantil de Pregrado, Grado y Posgrado” de la Secretaría Académica, Dirección de Información Académica e Investigación. Dirección de Grado/UNSAM.

11. Las denuncias y consultas se realizan en la Consejería de Atención a la Violencia de Género y Sexualidades, espacio que pertenece a la DGyDS 1 . El mismo funciona desde el año 2014 y aplica el protocolo de atención para situaciones de discriminación y violencia de género en la UNSAM.

12. Durante el año 2018 ha habido una creciente visibilización del colectivo de mujeres sobre situaciones de abuso y violencia que experimentaron a lo largo de su vida por parte de varones, a través de escraches en redes sociales. Este tema de gran relevancia excede el objetivo de este artículo y será trabajado en futuras publicaciones.

13. El protocolo mencionado se encuentra disponible en: http://www.unsam.edu.ar/secretarias/academica/dgyds.asp\#tab s-2

14. Posteriormente, se sumaron la Universidad de Buenos Aires (2015), la Universidad Nacional del Centro de la Provincia de Buenos Aires (2016), la Universidad Nacional de Misiones (2016), la Universidad Nacional de José C. Paz (2016) y la Universidad Nacional de Quilmes (2016). Y, los protocolos en Facultades: Facultad de Humanidades y Ciencias Sociales de la Universidad Nacional de la Patagonia San Juan Bosco - FHCS - UNPSJB (Comodoro Rivadavia -Provincia de Chubut), Facultades de Humanidades / Derecho / Ciencias Políticas y Relaciones Internacionales de la Universidad 
Nacional de Rosario - UNR (Rosario, Provincia de Santa Fe); Facultad de Trabajo Social de la Universidad Nacional de Entre Ríos - UNER (Paraná), Facultad de Humanidades y Ciencias Sociales, de la Universidad Nacional de Misiones UNaM (2016). Otras tantas ya se encuentran en proceso de elaboración.

15. Al día de la fecha, la Argentina cuenta con 55 universidades nacionales distribuidas en todo el territorio nacional.

16. Eldiario.es. "El tribunal reconoce que la violencia machista es un delito contra la seguridad pública". Enero $12,2017$. Recuperado de http://m.eldiario.es/andalucia/TSJA-determina-sentencia-violencia-seguridad_0_600890453.html

17. El nombre de la ley hace referencia al feminicidio de Micaela García, joven de 21 años quien fue violada y asesinada a la salida de un local bailable, en Gualeguay, por un varón que ya tenía antecedentes de ataques sexuales.

18. Se considera violencia física aquella que se emplea contra el cuerpo de la mujer produciendo dolor, daño o riesgo de hacerlo, y cualquier otra forma de maltrato o agresión que afecte su integridad física; la violencia sexual es cualquier acción que implique la vulneración en todas sus formas, con o sin acceso genital, del derecho de la mujer a decidir voluntariamente acerca de su vida sexual o reproductiva.

19. En el año 1999 el código penal argentino modificó el tipo "delitos contra la honestidad" por el de "delitos contra la integridad sexual”. Se sustituyó el artículo 119 del Código Penal, por el siguiente texto: "Será reprimido con reclusión o prisión de seis meses a cuatro años el que abusare sexualmente de persona de uno u otro sexo cuando, ésta fuera menor de trece años o cuando mediare violencia, amenaza, abuso coactivo o intimidatorio de una relación de dependencia, de autoridad, o de poder, o aprovechándose de que la víctima por cualquier causa no haya podido consentir libremente la acción”. En el año 2014 se introdujeron nuevos agravantes, para más información cotejar http://servicios.infoleg.gob.a r/infolegInternet/anexos/15000-19999/16546/texact.htm 